Rabaska

Revue d'ethnologie de l'Amérique française

\title{
Laboratoire de muséologie et d'ingénierie de la culture
}

\section{Philippe Dubé}

Volume 6, 2008

URI : https://id.erudit.org/iderudit/000174ar

DOI : https://doi.org/10.7202/000174ar

Aller au sommaire du numéro

Éditeur(s)

Société québécoise d'ethnologie

ISSN

1703-7433 (imprimé)

1916-7350 (numérique)

Découvrir la revue

Citer ce document

Dubé, P. (2008). Laboratoire de muséologie et d'ingénierie de la culture.

Rabaska, 6, 246-250. https://doi.org/10.7202/000174ar d'utilisation que vous pouvez consulter en ligne.

https://apropos.erudit.org/fr/usagers/politique-dutilisation/ 


\section{L'enseignement et la diffusion de la recherche}

En plus du cours d'été in situ à Percé, la titulaire de la Chaire donne des cours théoriques à l'École d'architecture et supervise des ateliers et des travaux pratiques, et ce, autant au baccalauréat qu'à la maîtrise. Des articles dans des revues et dans diverses publications scientifiques permettent de renseigner la communauté scientifique ainsi que la population en général sur les travaux menés par la Chaire. Ces informations sont accessibles sur le portail de la Chaire : www.crcprb.chaire.ulaval.ca. La Chaire regroupe actuellement une dizaine de jeunes chercheurs, un professionnel de la recherche et un collaborateur attitré, François Dufaux, architecte et chargé d'enseignement à l'École d'architecture de l'Université Laval.

Tania Martin et Diane Audy

\section{Laboratoire de muséologie et d'ingénierie de la culture}

Pavillon Casault, local 3545

Université Laval

Québec (Québec) G1K 7P4
Téléphone : (418) 656-2131, poste 3822 Courriel : Philippe.Dube@hst.ulaval.ca

Toile : www.lamic.ulaval.ca

Le LAMIC est un laboratoire de muséologie implanté au cœur de l'ancien Centre muséographique de l'Université Laval. Il est désormais opératoire, suite à une phase intensive de construction, rénovation et installation qui s'est déroulée de septembre 2006 à mai 2007. Notre équipe a requalifié le Centre muséographique en un lieu de recherche permettant de mesurer l'expérience muséale sous toutes ses formes : diffusion, éducation et conservation. L'approche canadienne de la muséologie contribue à la renommée internationale de nos savoir-faire culturels. Nous souhaitons permettre tant au milieu professionnel qu'à celui de la formation universitaire de faire des recherches approfondies qu'ils ne peuvent mener faute d'infrastructure. À l'aide d'un équipement technologique qui autorise, en termes mesurables, l'examen de l'expérience muséale, nous comptons objectiver l'appréciation des visiteurs et fournir des données précises aux acteurs culturels dans ce domaine effervescent.

\section{L'an 2 du LAMIC}

L'année qui vient de se terminer (juin 2008) a été majeure pour l'essor du Laboratoire de muséologie et d'ingénierie de la culture. D'abord, du point de 
vue de la recherche, plusieurs chercheurs ont pu se familiariser avec l'infrastructure et les équipements par le biais de divers projets. Puis, du point de vue de l'aménagement, le centre de documentation a été installé au cours de l'été 2007. Enfin, de nombreux événements et activités ont ponctué la vie du LAMIC au cours de l'année : conférences, visites, exposition et lancement du portail (www.lamic.ulaval.ca).

\section{Au chapitre de la recherche}

Plusieurs chercheurs ont pu recourir à l'infrastructure et aux équipements pour divers projets et expérimentations. Yves Bergeron (UQȦM), chercheur régulier au LAmic, et François Mairesse du Musée royal de Mariemont (Belgique) ont poursuivi cette année leurs travaux pour le projet Mémoires de la muséologie, qui veut rassembler et conserver les témoignages de conservateurs et muséologues majeurs de l'Europe et de l'Amérique du Nord. Les deux chercheurs réalisent donc une série d'entrevues avec les acteurs de la muséologie contemporaine. Geoffrey Edwards (Laval), chercheur régulier au LAMIC, a réalisé, au printemps 2007, des expérimentations pour une future exposition explorant la multi-ethnicité au cœur des villes modernes et la notion de périphérie. Cette dernière s'est tenue à Düsseldorf (Allemagne) en mai 2008. Marie-Christiane Mathieu (Laval), chercheur associé et artiste, s'est penchée sur la réalisation de sculptures sonores avec GPS, accompagnée entre autres par l'infrastructure du LAMIC. Un autre créateur, le dramaturge et scénographe Daniel Danis (Prix du Gouverneur général 2008), s'est associé au LAMIC pour des expérimentations sur l'écriture numérique, en intégrant des notions d'animation et de modélisation 3D. La collaboration s'est poursuivie sur plusieurs mois de l'année. Pour l'année 2007-2008, notons aussi la poursuite des travaux d'analyse en tracéométrie en vue des études doctorales de Marie-Michelle Dionne. Son directeur de recherche et chercheur régulier au LAMIC, Jacques Chabot (Laval), travaille quant à lui à l'élaboration d'une exposition archéologique sur les obsidiennes arméniennes.

Plusieurs étudiants ont été accueillis au Laboratoire, provenant de disciplines variées : théâtre (2), ingénierie (1+ équipe de Sherbrooke), robotique (1), géomatique (1).

Le LAMIC est également une infrastructure d'expérimentations importante pour des partenaires tels que les institutions muséales. Par exemple, le Musée de la civilisation a procédé à une batterie de tests, en avril 2008, pour l'installation du " puits » conçu par Franco Dragone dans le cadre de son «Potager des visionnaires » de l'été 2008. Le concepteur lui-même a été présent afin de valider sur place l'expérimentation concluante de son installation. Nous croyons qu'il s'agit là d'un pas important vers une collaboration intégrée avec le milieu muséal. 
Notre implantation aura permis des raccordements extrêmement vivaces avec d'autres laboratoires du seul fait que nous constituions, à partir de mai 2007, une nouvelle plate-forme dotée d'équipement reconnu comme étant performant et qui devenait dans ce contexte une monnaie d'échange avec les autres entités. Pour donner un seul exemple local de ce constat, nous n'avons qu'à citer les liens étroits qui existent maintenant à l'Université Laval entre le groupe REGARD (géomatique), le Laboratoire d'informatique cognitive, le Laboratoire de vision numérique et système, le Laboratoire de robotique et le Laboratoire des nouvelles technologies de l'information liées à la scène et au son (LANTISS). Toutes ces unités, sans perdre leur rattachement disciplinaire d'origine, œuvrent maintenant en association intime avec nous, d'une façon ou de l'autre (prêt d'équipement, collaborations de chercheurs, location d'espace, etc.). De plus à l'étranger, deux collaborations interlaboratoires se confirment à travers nos liens de plus en plus étroits avec le Centre d'étude et de recherche sur le lien social (CERLIS, Paris V) et le Laboratoire culture et communication (Université d'Avignon). Nous devons aussi signaler les efforts faits présentement afin de rallier à notre dynamique locale d'autres acteurs, notamment en Belgique (Liège et Musée royal de Mariemont), au Portugal (Université Lusophone de Lisbonne) et en Espagne (Universidad de Oviedo et le Goupe Mirar).

\section{Au chapitre de l'aménagement}

Pour compléter l'aménagement de l'espace-laboratoire, il ne manquait, l'année dernière, que l'installation du Centre de documentation. Aménagé en juillet et août 2007, il est depuis lors accessible sur demande aux chercheurs et aux étudiants. Pourvu de plus de 6000 documents, dont des ouvrages, des documents de référence, des catalogues d'expositions et des périodiques, on y retrouve également des affiches, des dépliants et une collection de milliers de cartons d'invitation à des événements muséaux. De plus, notons l'achèvement de l'inventaire des équipements du Laboratoire en septembre 2007, ce qui nous permet d'avoir une vue globale de nos capacités et potentialités.

\section{Au chapitre des activités}

L'événement majeur de l'année a été le lancement du site électronique du LAMIC le 6 mai 2008, dans le cadre de la visite des participants à deux colloques en muséologie du $76^{\mathrm{e}}$ congrès de l'AcFAs : Déjà plus, déjà là : la présentification et l'institution culturelle et La scénographie d'exposition aujourd'hui : crise et critique d'une fonction en mutation. La soixantaine de visiteurs a eu droit à un circuit de visite-démonstration des ateliers du LAMIC (captation, visualisation et spatialisation) avant le lancement officiel du site. Ce lancement 
s'est tenu en présence de monsieur Michel Audet, délégué du Québec à l'Unesco à Paris, de la directrice de l'Itis, madame Diane Poulin, et de monsieur Denis Mayrand, adjoint au vice-recteur à la recherche et à la création à l'Université Laval. Par la même occasion, un dépliant présentant éloquemment notre infrastructure de recherche en muséologie expérimentale a été divulgué. Conçu avec la collaboration de la Direction des affaires publiques et le Vice-rectorat à la recherche et à la création de l'Université Laval, ce dépliant constitue un très bon outil de promotion et de communication.

Un cycle de conférences s'est tenu de septembre 2007 à mars 2008. Onze professionnels et chercheurs du milieu de la muséologie sont intervenus sur des sujets divers tels que la conception d'exposition, la gestion et le financement de projets culturels, la théâtralité et le musée, la notion de périphérie : Victor Thibodeau (Université Laval), Monique Lippé (Musée de la civilisation), Christine Cheyrou et Mélanie Girard (Musée des Ursulines), Annette Viel, Michel Barry (Parcs Canada), Sylvie Royer (Centre Materia), Jennifer Boyes-Manseau (Musée canadien des civilisations), Dany Brown (Musée de la civilisation), Marie-Louise Bourbeau et Geoffrey Edwards (Université Laval).

Le projet d'un étudiant au DESs en muséologie s'est concrétisé grâce à notre laboratoire de spatialisation, l'ExpoLab. Il a en effet pu y monter une exposition de ses cuvres (des livres d'artistes), en passant par toutes les étapes de réalisation d'une exposition. "Photographie, livre et délire. Exposition des livres d'artiste de Guy Cusson » s'est déroulée à l'ExpoLab du LAMIC du 25 avril au $1^{\text {er }}$ mai 2008.

Plusieurs groupes sont venus visiter les installations du LAMIC au cours de l'année, dont les responsables de la Division du patrimoine des Forces armées canadiennes, des chercheurs étrangers du colloque du CÉLAT organisé par Jocelyn Létourneau, une délégation internationale du congrès 2007 de la SMQ, une délégation sénégalaise du Ministère de l'Éducation nationale et de l'Université Gaston-Berger de Saint-Louis, l'équipe de direction d'UBISOFT Québec et des participants au congrès de l'Association des archivistes du Québec.

De plus, le Lamic s'est investi dans un projet d'exposition intitulé "Québec s'écrit... ». La Bibliothèque de l'Université Laval, commanditaire et organisatrice de l'exposition, a ainsi bénéficié du soutien du LAMIC par le biais de son directeur pour l'appui scientifique et de son polytechnicien pour l'appui technique. Deux diplômées en muséologie, Virginie Benjamin et Lydia Bhérer-Vidal, associées au LAMIC ont également agi à titre de chargées de projet pour cette exposition. 


\section{Projets en développement}

Voici la liste de ces projets : projet de la BuLLE (avec la Bibliothèque générale de l'Université Laval) ; projet de recherche bilatérale (Acc. can.-fr.) sur les collections universitaires ; projet de télé-collaboration avec André Gob (Liège) et François Mairesse (Musée royal de Mariemont) - Office de coopération Québec-Bruxelles-Wallonie; projet de Land Museum au Musée de Masteuiash (Géraldine Laurendeau) ; projet de connexion des communautés autochtones du Québec dotées d'un musée (Stations de Télé-Présence/SAT) ; projet de résidence d'artistes avec la Chambre blanche; projet de recherche avec le Cerlis (Paris V) "Réception comme Création»; projet de regroupement d'intérêt scientifique : PENSEE; projet sur la culture scientifique et la jeunesse francophone ; projet de conférence internationale en 2010 (RCIP/ CHIN); projet de recherche avec le Forum canadien de recherche publique sur le patrimoine (www.patrimoine-canada.ca).

PhilipPe Dubé

\section{Conseil québécois du patrimoine vivant}

310 boul. Langelier, bureau 242

Québec (Québec) G1K 5N3

Téléphone : (418) 524-9090

Courriel : cqpv@cqpv.qc.ca

Toile : www.cqpv.qc.ca

Entre la poursuite de ses missions de concertation et de regroupement, la mise en œuvre d'un important chantier sur la professionnalisation des pratiques en patrimoine vivant, lui-même émanant de la poursuite des projets de formation professionnelle, et l'actualité gouvernementale induite par le dépôt du livre vert sur la refonte de la Loi sur les biens culturels du Québec, l'année du Conseil québécois du patrimoine vivant $(\mathrm{CQPV})$ a été fertile en développements de toutes sortes.

\section{Concertation et formation professionnelle}

La table de concertation sur la danse traditionnelle qui avait mis en place le premier stage de formation professionnelle pour les enseignants de la danse s'est dorénavant incorporée et est devenue une association autonome, Danse traditionnelle Québec (DтQ). Le secteur s'en est trouvé renforcé d'autant : loin de constituer une scission, la naissance de DTQ a permis de préciser le partenariat entre le $\mathrm{CQPV}$ et le milieu en fonction des attentes et des besoins spécifiques de chaque type de pratique. Ainsi, pour la danse, après le succès 